\title{
Improved 2-D Vector Field Reconstruction using Virtual Sensors and the Radon Transform
}

\author{
Archontis Giannakidis*, Leonidas Kotoulas* and Maria Petrou**
}

\begin{abstract}
This paper describes a method that allows one to recover both components of a 2 -D vector field based on boundary information only, by solving a system of linear equations. The analysis is carried out in the digital domain and takes advantage of the redundancy in the boundary data, since these may be viewed as weighted sums of the local vector field's Cartesian components. Furthermore, a sampling of lines is used in order to combine the available measurements along continuous tracing lines with the digitised 2-D space where the solution is sought. A significant enhancement in the performance of the proposed algorithm is achieved by using, apart from real data, also boundary data obtained at virtual sensors. The potential of the proposed method is demonstrated by presenting an example of vector field reconstruction.
\end{abstract}

\section{INTRODUCTION}

In recent years Hough transform [1] and the related Radon transform [2] have found widespread use. Theoretical ideas found in Radon's early work provide the mathematical basis for conventional tomographic reconstruction, very common in medical imaging. Functions that are reconstructed by using traditional tomography are scalar functions describing e.g., absorption or scattering coefficients. However, over the last few decades there has been a growing demand for similar techniques that would perform tomographic reconstruction of a vector field, rather than a scalar one, when having integral information. The problem of recovering a vector field from its line integrals has generally been regarded as an 'underdetermined' problem. This seems to be clear from the fact that a scalar function is determined uniquely from its Radon transform, whereas a vector field requires two (in 2D) or three (in 3-D) component functions to be determined. ${ }^{1}$

\section{LITERATURE SURVEY}

During the short history of 2-D vector field tomography, many attempted to solve the reconstruction problem [3], [4], [5], but the result has always been the same: only one component of the vector field could be recovered from the tomographic measurements. The component that could be recovered was either the curl-free (irrotational) part or the divergence-free (solenoidal) part, depending on the physical principle of the measurements, namely the relation between the obtained set of measurements and the investigated vector

This work was supported by the "Integrated Electronics" portfolio grant. *A. Giannakidis and L. Kotoulas are with Faculty of Engineering and Physical Sciences, University of Surrey, Guildford, GU2 7XH, UK a.giannakidis@surrey.ac.uk, 1.kotoulas@surrey.ac.uk

**M. Petrou is with the Department of Electrical and Electronic Engineering, Imperial College, Exhibition Road, South Kensington, London, SW7 2AZ, UK maria.petrou@imperial.ac.uk

${ }^{1}$ This analysis deals only with vector fields that have two components. field. One possible solution to this problem would be to collect data using both types of interaction between the measurements and the examined vector field for every application. Indeed, such an amount of information would be sufficient to allow for a full reconstruction of the vector field as Braun and Hauck demonstrated in [4]. Unfortunately, there are only very few specialized applications (mainly in optics), where it is physically realizable to have all these measurements available. Moreover, as Norton showed in [3] we may have a full reconstruction based only on longitudinal measurements, as long as, apart from the longitudinal measurements, supplementary information about the vector field, especially boundary conditions and a priori information about the source distribution, is available as well. However, all the work accomplished in vector field tomography has been carried out in the continuous domain and it does not take into consideration the redundancy in the data used to reconstruct the field. Although along each line we measure only one component of the field (along or perpendicular to the direction of the line), we may use many line orientations passing through every point and then view their recordings as weighted sums of the local vector field's Cartesian components. Besides, in the discrete domain, we want to recover the field only at sampling points. We may exploit then the redundancy in the data to recover the vector field at all sampling points of the 2-D domain. We shall discuss this method next.

\section{THE PROPOSED METHODOLOGY}

We formulate the vector field reconstruction problem in terms of Cartesian vector components required at sampling points of a 2-D domain. We wish to recover all components of a vector field $\overline{\mathbf{f}}(x, y)$ based only on integral data. In order to achieve the full vector field recovery, we exploit the redundancy in the integral data, since these data may be viewed as weighted versions of the local vector field's Cartesian components. The whole treatment in this section is performed in the digital domain. The bounded 2-D domain, within which we want to recover the vector field, is divided into tiles of finite size. The values of the components of the vector field in every such tile are the values of the components of this field at the centre of the tile, namely the sampling point. Similarly, the available redundant lineintegral (boundary) data are not in the continuous domain either. There is only a finite number of pairs of points, that reside on the boundary of the 2-D domain, where we may obtain the line-integral data. These points are assumed to be the locations of ideal point sensors. Consequently, the 
solution to the reconstruction problem will be based only on these line-integral data. The ultimate objective of this analysis is to recover both components of the vector field at every sampling point of the bounded 2-D domain by solving a system of linear equations. In order to form this system of linear equations we assume that our sensors integrate only the component of the field projected on the line. Then, every line-integral $\left(J_{i}\right)$ that is available gives rise to an equation. Hence, by using all available line-integrals we obtain the required system of linear equations, the solution of which is expected to give all the components of the vector field at all sampling points of the 2-D domain. It must be noted that we have two unknowns per sampling point of the 2-D space, namely the components $\left(f_{x}, f_{y}\right)$ of the vector field. As the number of the available line-integrals (equations) is determined by the number of boundary points, where we make the measurements, we may select the value of the latter so that the number of linear equations is twice the number of sampling points of the 2-D space. If more equations are available, the solution will be obtained in the least-square error sense.

In order to combine the measurements along continuous tracing lines with the digitisation of the 2-D space where the solution is sought, we consider regular sampling points along the tracing lines. The sampling process we use is similar to the one described in [6]. Let us assume that we have the digitised square 2-D domain shown in Fig. 1 and we wish to recover vector field $\overline{\mathbf{f}}(x, y)$ at the centre of every tile of this space. The length of each side of the square domain is taken to be equal to $2 L$ and the origin of the axes of the coordinate system is chosen to be at the centre of the domain. The size of the tiles with which we sample the 2-D domain is $P$, so that $2 L / P$ is an integer. Let us consider a line segment $A B$ crossing this domain as shown in Fig. 1. The coordinates

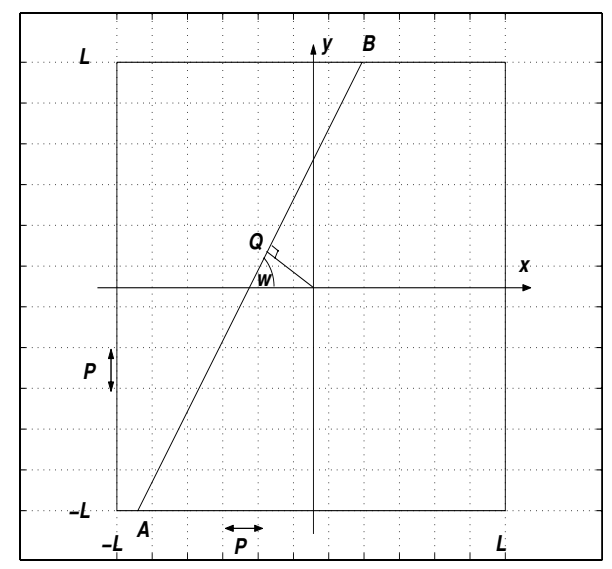

Fig. 1. A line segment between two boundary points, $A$ and $B$, that goes through a digital square domain of side length equal to $2 L$. The angle between the line segment and the positive direction of the $x$-axis is $w$. The size of the tiles, with which we sample the 2-D space, is $P$. Point $Q$ is the foot of the normal of the considered line segment from the origin of the axes.

of points $A$ and $B$ are $\left(x_{A}, y_{A}\right)$ and $\left(x_{B}, y_{B}\right)$, respectively.
Therefore, the equation of line $A B$ is

$$
y=\lambda x+\beta
$$

where

$$
\lambda \equiv \frac{y_{B}-y_{A}}{x_{B}-x_{A}} \quad \text { and } \quad \beta \equiv y_{A}-\frac{y_{B}-y_{A}}{x_{B}-x_{A}} x_{A}
$$

The unit vector $\hat{\mathbf{s}}$ along the line (and with direction from $A$ to $B$ ) is:

$$
\hat{\mathbf{s}}=\cos w \hat{\mathbf{x}}+\sin w \hat{\mathbf{y}}
$$

where $w=\arctan \lambda$ is the angle between the line and the positive direction of the $x$-axis (Fig. 1). The next step is to perform a sampling of the line segment. The starting point of this sampling will be the foot of the normal of this line from the origin of the axes (point $Q$ in Fig. 1). The coordinates of the starting point $Q$ for the calculations along the line are:

$$
x_{Q}=-\frac{\beta}{\left(\lambda+\frac{1}{\lambda}\right)}, \quad y_{Q}=-\frac{1}{\lambda} x_{Q}
$$

The sampling along the line section will be performed on either side of $Q$ and we assume that the sampling step is $\Delta s$. The maximum number of sampling intervals that we can fit in this line section is determined by the intersection points between the line and the border of the 2-D domain. The distances between the starting point $Q$ and intersection points $A$ and $B$ are $d_{A}$ and $d_{B}$ respectively. Consequently, the numbers $l_{A}$ and $l_{B}$ of $\Delta s$, that we may fit in the line segment between the foot of the normal, $Q$, and the boundary points $A$ and $B$ are, respectively:

$$
l_{A}=\left\lfloor\frac{d_{A}}{\Delta s}\right\rfloor, \quad l_{B}=\left\lfloor\frac{d_{B}}{\Delta s}\right\rfloor
$$

where $\lfloor\cdot\rfloor$ is the symbol for the floor operator. Therefore, the sampling points we shall consider along the line segment will have coordinates

$x_{l}=x_{Q}+l x_{i n c}, \quad y_{l}=y_{Q}+l y_{i n c}$ for $l \in\left[-l_{A}, l_{B}\right]$

where the increments $x_{i n c}$ and $y_{i n c}$ of the coordinates between successive sampling points are given by:

$$
x_{i n c}=\Delta s \cos w, \quad y_{i n c}=\Delta s \sin w
$$

The total number of sampling points along the line segment is $l_{A}+l_{B}+1$. After having worked out the coordinates of the sampling points of the line, we must assign them values from the vector field. We use nearest neighbour interpolation for that. Hence, we need to determine for each sampling point of the line, the tile the centre point of which is its nearest neighbour. For this purpose, we use the integer coordinates $(i, j)$ with $i, j=1, \ldots, \frac{2 L}{P}$, of each tile of the 2 D domain.Then, the tile $(i, j)$ that corresponds to a sampling point $\left(x_{l}, y_{l}\right)$ is identified by using the formulae:

$$
i=\left\lceil\frac{x_{l}+L}{P}\right\rceil, \quad j=\left\lceil\frac{y_{l}+L}{P}\right\rceil
$$

where $\lceil\cdot\rceil$ is the ceiling operator.

The next step is to form the equation that corresponds to the line-integral measurement $J_{i}$ between points $A$ and $B$. 
This may be achieved by considering the sampling points of the line that we obtained as the centres of linear segments of length $\Delta s$, apart from the sampling points with $l=-l_{A}$ and $l=l_{B}$ which are special cases. We then convert the integral into a sum by projecting the value of the field at each sampling point of the line onto the vector that represents the corresponding integrating element:

$$
J_{i}=\sum_{l=-l_{A}+1}^{l_{B}-1} \overline{\mathbf{f}}_{l} \cdot \overline{\Delta \mathbf{s}}+\overline{\mathbf{f}}_{l A} \cdot \hat{\mathbf{s}} \Delta_{A}+\overline{\mathbf{f}}_{l B} \cdot \hat{\mathbf{s}} \Delta_{B}
$$

where $\overline{\mathbf{f}}_{l}, \overline{\mathbf{f}}_{l A}$ and $\overline{\mathbf{f}}_{l B}$ are the vector field values at sampling points $l, l_{A}$ and $l_{B}$ respectively, $\overline{\Delta \mathbf{s}}=\Delta s \hat{\mathbf{s}}$, and

$$
\Delta_{A}=\frac{\Delta s}{2}+d_{l A}, \quad \Delta_{B}=\frac{\Delta s}{2}+d_{l B}
$$

where $d_{l A}$ is the distance between the sampling point with $l=-l_{A}$ and the boundary point $\mathrm{A}$, whereas $d_{l B}$ is the distance between the sampling point with $l=l_{B}$ and the boundary point $\mathrm{B}$.

The method described above is based on linear algebra. This approach formulates the tomographic reconstruction problem in terms of a system of linear equations. However, there is a duality between the Radon transform scheme and this matrix formalism. Hence, the solution of the above described system of linear equations is equivalent to inverting the vectorial Radon transform. According to the theory of the Radon transform [7], the exact image reconstruction requires projection data as a continuous function of two variables, normally designated as the radial and angular coordinates. However, in practice this is never available and projections are measured with only limited resolution. Moreover, when using discrete approximations, necessary requirements to produce results with the accuracy desired in medical imaging is to have a large number of projections (ie., adequately dense sampling of the Radon domain parameters) and, also, substantially uniform distribution of Radon data. In this paper, in order our discrete approach to satisfy better the above described criteria and, also, to be closer to the ideal continuous case, we have increased the number of the boundary data, by adding virtual sensors. The values of the integral data at these sensors are calculated from the values at the nearby sensors by using linear interpolation. Experimental results of this technique are discussed in Section IV.

\section{AN EXAMPLE: ELECTRIC FIELD IMAGING}

In this section we consider the case where the vector field that we want to recover is the electric field created by a static charge. There are many ways to recover the electric field from boundary data. However, here we use the electric field only to demonstrate our method. In order to avoid problems with singularities, we treat the case where the source of the vector field that we aim to recover is outside the bounded 2-D area. In a real physical system, we do not expect to have to deal with real singularities. Again, we stress that the problem we solve is intentionally kept simple in order to demonstrate the method. So, instead of using a realistic version of Coulomb's law where the source of the field is finite, we place the source outside the domain of interest and make it infinitesimally small. For this electric field recovery, the data we shall rely entirely on are line-integral data taken at the border of the 2-D area. A fundamental property regarding the electric field, resulting from the fact that the electric field created by static charges is irrotational, is that the voltage difference between any two stated points, $A$ and $B$, is given by the integral of the projections of the electric field onto any curve uniting these two points. By letting points $A$ and $B$ to range over the boundary of a domain within which we want to recover the electric field and, also, by restricting the integration curves to be the line sections uniting any two such points, we may deduce that every voltage difference between any two points that reside on the boundary of a domain may be treated as a lineintegral when trying to recover the electric field within this domain. We may also say that these voltage differences give the vectorial Radon transform of the electric field. It must be noted that the electric field is irrotational, so according to [3], transversal measurements only would be enough to recover this field. However, the only realizable measurements for this application are longitudinal.

To exemplify the theory, two simulations were performed. The geometry we employed for the digital domain is a square domain of size $2 L \times 2 L$ where $2 L=11$ and the tile size $P$ was taken equal to 1 . Hence, the domain consists of $121(=11 \times 11)$ tiles. The location of the source was taken at $(16,18)$. The electric field reconstruction was based only on a number of voltage differences obtained between points that lay on the boundary of this domain. In the first simulation, we assumed that there are ideal point sensors (electrodes) regularly placed in known positions of the border of the domain (these are the middle points of the boundary edge of all boundary tiles) and data from these points were used only. Hence, we used 11 sensors in every side of the boundary of the square domain. In all these known and predetermined points we acquired the simulated potentials by using Coulomb's law. After that, we considered all possible voltage differences between any pair of these boundary points and we formed the system of linear equations according to the description of the previous section. However, voltage differences between boundary points that reside in the same side of the square boundary were not used. Moreover, the sampling step along the line segments, that unite boundary points, was selected to be equal to 0.0009 of the tile size. The number of equations (available voltage differences) of the system was 726 , whereas the number of the unknowns $\left(E_{x}, E_{y}\right.$ components for every tile of the domain) was $242(=11 \times 11 \times 2)$. For the second simulation, we also inserted virtual sensors, one in between every two real sensors. Then, the vector field reconstruction was also based on the interpolated voltages at these virtual sensors. The employment of virtual sensors resulted in having 21 sensors in total in every side of the boundary and, also, the number of available voltage differences was 2646 .

The reconstruction results, namely the solution of the overdetermined system of linear equations for the two simulations 

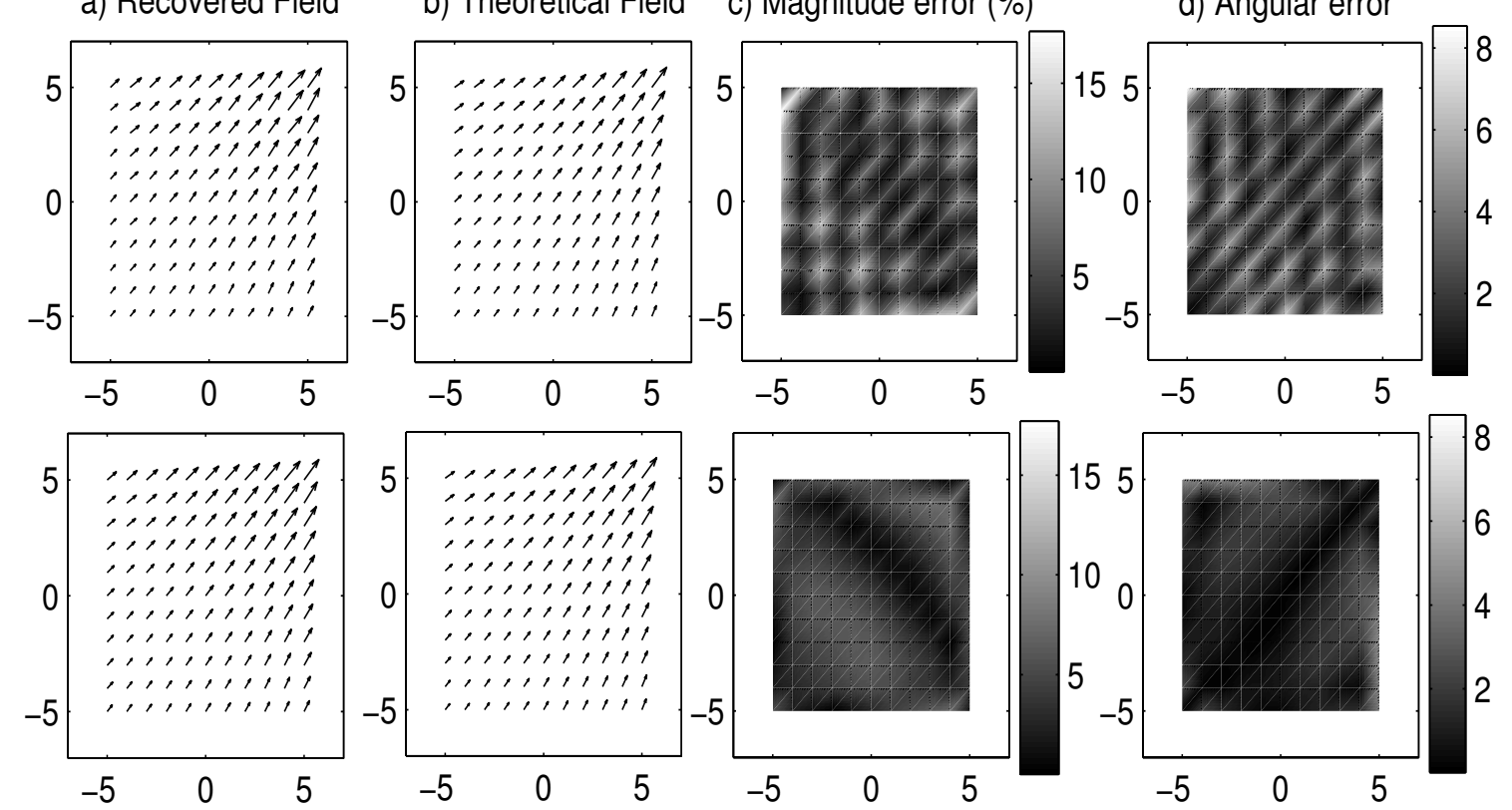

Fig. 2. Simulation results for the case where the location of the source of the electric field is at $(16,18)$ when (top) only boundary data from the real sensors are used, and (bottom) data from the virtual sensors are also used: a) The recovered vector field (solution of the system of linear equations), b) The electric field as computed from Coulomb's law, c) The relative error in magnitude, d) The absolute angular error (in degrees).

(without and with the use of virtual sensors), are shown in Fig. 2. For the sake of comparison, Fig. 2 also depicts the respective electric fields that are obtained by using directly the theoretical Coulomb's law. Moreover, in Fig. 2 the relative differences between the magnitudes of the two vector fields (i.e., the absolute values of the differences between the magnitudes of the reconstructed field and the theoretical field as acquired by Coulomb's law divided by the theoretical magnitude) for both simulations can be seen, as well as the angular differences (in degrees) between the reconstructed vector field values and the theoretical ones. By inspection of Fig. 2, we may see that the introduction of the virtual sensors, resulted in a much better vector field reconstruction. In particular, it was found that the average squared error of the magnitude per pixel was reduced by $59 \%$ after inserting the virtual sensors, whereas the average squared angular error dropped by $68 \%$.

\section{Summary And CONClusions}

In this paper, the vector field tomography problem was discussed. In previous attempts to map integral measurements obtained along tracing lines onto a vector field, conventional (scalar) tomography theory had invariably been applied [3], [8]: this had led to an under-determined problem. However, in this paper a new analysis was presented that aimed at the recovery of all components of a field at the sampling points of a 2-D digitised bounded domain by solving a system of linear equations. The reconstruction was based only on boundary data. We took advantage of the redundancy in boundary data with a view to recovering the vector field fully, since these data may be seen as weighted sums of the local vector field's Cartesian components. In the approach we followed, emphasis was placed on the use of sampling lines with a view to achieving better accuracy in expressing the integrals, that refer to the redundant available measurements along continuous tracing lines, in terms of the finite number of the values of the vector field at the centres of tiles. In order to enhance further the performance of the proposed algorithm we used interpolated boundary data obtained at virtual sensors. This heuristic resulted in a significant reduction of both the angular and magnitude recovery error. The reconstruction results we obtained for a number of simulations demonstrate that we may achieve full recovery of a vector field through this approach.

\section{REFERENCES}

[1] P.V.C. Hough, A Method and Means for Recognizing Complex Patterns, US Patent, vol. 3, no. 069, 1962, pp. 654.

[2] J. Radon, Uber die Bestimmung von Funkionen durch ihre Integralwerte Längs Gewisse Mannigfaltigkeiten, Berichte der Sächsischen Akademie der Wissenschaften (Leipzig), Mathematische - Physikalische Klasse, vol. 69, 1917, pp 262-277.

[3] S.J. Norton, Unique Tomographic Reconstruction of Vector Fields Using Boundary Data, IEEE Transactions on Image Processing, vol. 1, no. 3, 1992, pp 406-412.

[4] H. Braun and A. Hauck, Tomographic Reconstruction of Vector Fields, IEEE Transactions on Signal Processing, vol. 39, no. 2, 1991, pp 464471.

[5] V.A. Sharafutdinov, Tomographic problem of photoelasticity, in Proceedings of the Society for Photo-Optical Instrumentation Engineers, SPIE, vol. 1843, 1992, pp 234-243.

[6] M. Petrou and F. Wang, A tutorial on the practical implementation of the trace transform, Ch. 11, Handbook of Texture Analysis, Mirmehdi, Xie, Suri, World Scientific, ISBN: 978-1-84816-115-3; 2008.

[7] S.R. Deans, The Radon Transform and Some of Its Applications, Wiley, New York; 1983.

[8] W. Munk and C. Wunsch, Observing the Ocean in the 1990s, Philosophical Transactions of the Royal Society of London. Series A, Mathematical and Physical Sciences, vol. 307, no. 1499, 1982, pp 439-464. 